\title{
Insight into the Interaction between the HIV-1 Integrase Inhibitor Elvitegravir and Bovine Serum Albumin: A Spectroscopic Study
}

\author{
Ali Saber Abdelhameed \\ Department of Pharmaceutical Chemistry, College of Pharmacy, King Saud University, P.O. Box 2457, Riyadh 11451, Saudi Arabia \\ Correspondence should be addressed to Ali Saber Abdelhameed; asaber@ksu.edu.sa
}

Received 2 February 2015; Revised 4 March 2015; Accepted 5 March 2015

Academic Editor: Rizwan Hasan Khan

Copyright (C) 2015 Ali Saber Abdelhameed. This is an open access article distributed under the Creative Commons Attribution License, which permits unrestricted use, distribution, and reproduction in any medium, provided the original work is properly cited.

\begin{abstract}
The interaction between the anti-HIV drug Elvitegravir (EVG) and bovine serum albumin (BSA) was investigated by fluorescence spectroscopy and UV-visible absorption spectra. The mechanism for quenching the fluorescence of BSA by EVG is discussed. It was found that EVG can quench the intrinsic fluorescence of BSA through a static quenching procedure. The quenching type, association constant, and number of binding sites were investigated. The binding constant of EVG with BSA was calculated at different temperatures based on fluorescence quenching results. The thermodynamic parameters $\Delta H^{\theta}, \Delta G^{\theta}$, and $\Delta S^{\theta}$ were determined. The positive $\Delta S^{\theta}$ and negative $\Delta H^{\theta}$ and $\Delta G^{\theta}$ values showed that a spontaneous interaction may involve both roles of hydrophobic interaction and hydrogen bonding. The interaction of BSA with EVG was also confirmed by UV absorption spectra. The average distance, $r$, between donor (BSA) and acceptor (EVG) was obtained according to Förster's theory of nonradiation energy transfer. Synchronous fluorescence and three-dimensional fluorescence spectra were used to investigate the conformational change of BSA molecules that occur upon addition of EVG and showed, upon binding, a possibility of increasing hydrophobicity around tryptophan residues of BSA.
\end{abstract}

\section{Introduction}

Serum albumins are the major soluble protein constituents of the circulatory systems, and they have many physiological functions $[1,2]$. They act as storage and transport carriers for various endogenous and exogenous compounds $[3,4]$. Therefore, interaction of drugs with and competition for the binding sites on albumins may strongly affect the distribution and elimination rate of the drugs as well as their action mechanism and pharmacodynamics. Hence, investigating the drug binding with albumins will facilitate interpretation of the metabolism and transport process of the drug and will help to elaborate the relationship between structures and functions of proteins. We have selected bovine serum albumin (BSA), for which association constants and binding sites are known for a number of compounds $[5,6]$. BSA and human serum albumin (HSA) display approximately $76 \%$ sequence homology $[7,8]$. Similar to other serum albumin, bovine serum albumin (BSA) is a multifunctional protein that possesses various physiological functions including binding, transport, and delivery of fatty acids $[9,10]$, porphyrins [11], bilirubin [12], and several other molecules. BSA is also responsible for certain binding aggregation and conformational dynamics in solution [13]. BSA has been extensively studied [14-16], and its molecular interactions with several molecules are often determined via spectroscopic techniques due to their high sensitivity, reliability, and ease of use [17-21]. On the other hand, integrase inhibitors are one of the more recently introduced classes of antiretroviral [22, 23]. Elvitegravir (EVG) (formerly GS-9137, JTK-303) (6-(3-Chloro-2-fluorobenzyl)1-[(2S)-1-hydroxy-3-methyl-2-butanyl]-7-methoxy-4oxo-1,4-dihydro-3-quinolinecarboxylic acid) (Figure 1) is a potent, boosted, once daily, HIV integrase inhibitor through the inhibition of DNA strand transfer [24, 25]. EVG has antiviral activity against wild-type and drug 


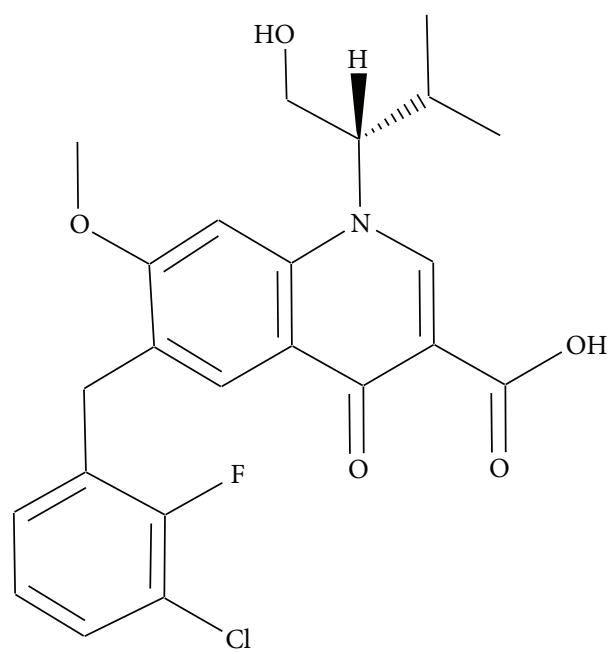

FIGURE 1: Chemical structure of EVG.

resistant strains of HIV [24]. EVG inhibits the process of viral DNA strand transfer with the greatest potency $50 \%$ maximal inhibitory concentration [IC50] of $<0.06 \mu \mathrm{mol} \cdot \mathrm{L}^{-1}$ in vitro [26], thereby preventing HIV DNA from being integrated into host chromosomes and blocking provirus formation and infection propagation [27]. The inhibition of strand transfer occurs via the binding of Elvitegravir to the integrase-viral DNA complex and the drug's subsequent interaction with the DNA and the essential magnesium ions within the active site of the integrase [22, 28]. EVG initially became available as part of a fixed-dose tablet (Stribild) in combination with emtricitabine, tenofovir disoproxil fumarate, and the pharmacokinetic boosting agent cobicistat in several countries, including North America and the EU [22]. Elvitegravir has also been approved in the EU and more recently (Sep. 2014) by the FDA as a single-agent tablet (Vitekta 85 or $150 \mathrm{mg}$ ) for use in combination with a ritonavirboosted protease inhibitor plus one or more antiretrovirals for treatment-experienced HIV-positive adults [29, 30]. With the use of antiretroviral drugs, several potential interactions have to be considered. In the current study, the EVG induced quenching of the intrinsic fluorescence of BSA was investigated. BSA consists of a chain of 580 amino acid residues composing a well-defined single polypeptide sequence, which contains three homologous $\alpha$-helix domains (I-III) $[31,32]$ and two tryptophan residues located at positions 134 and 212 of the chain [33]. The fluorescence intensity of BSA could be decreased by quenchers such as many drugs or small molecule organic compounds [34]. The changes of fluorescence or absorption spectra of BSA reflect the interaction of drugs and macromolecule protein. Extensive survey of the literature revealed that no reports studying EVG and serum albumin interactions were published. Hence, the current study is designed to study the interaction of EVG and BSA using fluorescence spectroscopy to explain the quenching type, the association constants, number of binding sites, and the basic thermodynamic parameters.

\section{Materials and Methods}

2.1. Materials. Elvitegravir (EVG; CAS 697761-98-1) was purchased from MedChemexpress (NJ, USA). Bovine serum albumin (BSA) was purchased from Techno Pharmchem (Bahadurgarh, Haryana, India). HPLC grade methanol was purchased from BDH laboratory supplies (Poole, UK). Potassium dihydrogen orthophosphate anhydrous was purchased from Central drug house Ltd. (New Delhi, India). Sodium phosphate dibasic, anhydrous, was purchased from Bio Basic Inc. (NY USA). Extra pure sodium chloride purchased from Loba Chemie Pvt. Ltd. (Mumbai, India) Potassium chloride was purchased from WINLAB Ltd. (Leicestershire, UK). Ultrapure water of $18 \mu \Omega$ obtained from a Millipore Milli-Q UF-Plus purification system (Millipore, Bedford, MA, USA) was used throughout the study.

2.2. Apparatus. A Jasco FP-8200 spectrofluorometer (JASCO International Co. Ltd. Tokyo, Japan) was used for all the fluorescence measurement, with excitation and emission slits at $5 \mathrm{~nm}, \lambda_{\text {ex }}=280 \mathrm{~nm}$ and $1-\mathrm{cm}$ quartz cell. In the study, BSA was excited at $280 \mathrm{~nm}$ and monitored at $338 \mathrm{~nm}$. All absorption spectral recordings and absorbance measurements were performed on a Cary $50 \mathrm{UV}-\mathrm{Vis}$ spectrophotometer (Agilent Technologies, Santa Clara, CA, USA). All measurements were performed in 1X phosphate buffered saline (PBS buffer) $\mathrm{pH}$ 7.4; the $\mathrm{pH}$ was measured on an Adwa AD1030 pH-meter (ADWA Instruments Inc., Romania).

2.3. Sample Preparation. EVG standard solution was prepared in methanol yielding a final concentration of $1.0 \mathrm{mg} \mathrm{mL}^{-1}$. A volume of $1.0 \mathrm{~mL}$ stock solution was diluted $10 \mathrm{~mL}$ with methanol to give a $100 \mu \mathrm{g} \mathrm{mL}^{-1}$ concentration as a working standard solution. The working solution was further diluted with PBS buffer $\mathrm{pH} 7.4$ to produce a working standard solution of $20 \mu \mathrm{g} \mathrm{mL}^{-1}$.

Bovine serum albumin (BSA) solution was prepared in a concentration of $1.0 \mathrm{mg} \mathrm{mL}^{-1}$ by dissolving $0.1 \mathrm{~g}$ pure BSA in PBS buffer pH 7.4 and completing the volume to $100 \mathrm{~mL}$ and kept in the cool, dark. This solution was further diluted with PBS buffer $\mathrm{pH} 7.4$ to produce a working standard solution of $100 \mu \mathrm{g} \mathrm{mL}^{-1}$.

\subsection{Procedures}

2.4.1. EVG-BSA Interactions. Upon optimizing the concentrations of both analytes, the BSA concentration was fixed at $50 \mu \mathrm{g} \mathrm{mL}^{-1}$ with EVG concentration that ranged from 0.1 to $15 \mu \mathrm{g} \mathrm{mL}^{-1}$. Fluorescence measurements were performed at three temperatures $(288,298$, and $309 \mathrm{~K}$ ) in the range of $290-500 \mathrm{~nm}$ upon excitation at $280 \mathrm{~nm}$. The threedimensional fluorescence spectrum was performed using the initial excitation wavelength set at $240 \mathrm{~nm}$ up to $350 \mathrm{~nm}$ with an increment of $2 \mathrm{~nm}$. Emission wavelength was set between 220 and $600 \mathrm{~nm}$ and an increment of $0.5 \mathrm{~nm}$. Synchronous fluorescence was performed with a $\Delta \lambda$ values of 15 and 60 scanning between 260 and 500 with a $0.5 \mathrm{~nm}$ data interval. 
2.4.2. UV Measurements. The UV-vis spectra of BSA were recorded for the drug-free protein and upon addition of EVG scanning in the wavelength range of $220-500 \mathrm{~nm}$. BSA concentration was kept constant at $0.8 \mathrm{mg} \mathrm{mL}^{-1}$ while the drug concentration was 3.0, 5.0, 7.0, and $10.0 \mu \mathrm{g} \mathrm{mL}^{-1}$.

2.4.3. Energy Transfer between EVG and BSA. A concentration of $10.0 \mu \mathrm{g} \mathrm{mL}^{-1}$ of EVG was used for the absorption measurement in the range of $220-500 \mathrm{~nm}$. Meanwhile, BSA $\left(50 \mu \mathrm{g} \mathrm{mL}^{-1}\right)$ emission spectrum was determined in the wavelength range of 280-500 $\mathrm{nm}$. Hence, the energy transfer was computed in accordance to Förster's nonradiative energy transfer theory (FRET) [35] utilizing the overlapped UV absorption spectrum of EVG with the fluorescence emission spectrum of BSA.

\section{Results and Discussion}

3.1. Fluorescence Quenching of BSA Induced by EVG. Spectroscopic techniques, particularly fluorescence spectroscopy, are able to provide enormous amount of information on the ligand protein binding, such as type, mechanism and strength of the binding, and so forth. It has been previously established that quenching of fluorescence intensity, that is, fluorescence intensity reduction of a certain fluorophore, can occur via several mechanisms including but not limited to formation of a nonfluorescent complex and energy transfer, and so forth $[15,36,37]$. The fluorescence spectra of BSA in presence of different amounts of EVG were measured in the range of $288-500 \mathrm{~nm}$ upon excitation at $280 \mathrm{~nm}$. EVG led to a concentration dependent quenching of the intrinsic fluorescence of BSA (Figures 2 and 3) without changing the emission maximum but with a slight change in peak shape at higher EVG concentrations $\left(>10 \mu \mathrm{g} \mathrm{mL}^{-1}\right)$. These results indicated that an interaction between EVG and BSA is taking place [38].

Furthermore, the fluorescence quenching data were analyzed by the Stern-Volmer equation (1) [39] and LineweaverBurk equation (2) [40]. Consider

$$
\begin{gathered}
\frac{F_{0}}{F}=1+K_{\mathrm{SV}} Q=1+K_{q} \tau_{0} Q \\
\left(F_{0}-F\right)^{-1}=F_{0}{ }^{-1}+K_{\mathrm{LB}}{ }^{-1} F_{0}{ }^{-1} Q^{-1},
\end{gathered}
$$

where $F_{0}$ and $F$ are the steady-state fluorescence intensities of BSA in the absence and presence of EVG, respectively, $K_{\mathrm{SV}}$ the Stern-Volmer quenching constant and $Q$ is the concentration of quencher (EVG). $K_{q}$ is the quenching rate constant, $\tau_{0}$ is the average lifetime of the protein without the quencher and $K_{\mathrm{LB}}$ is the static quenching constant. Within certain concentration, the curve of $F_{0} / F$ versus $Q$ (Stern-Volmer curve) would be linear if the quenching type is single static or dynamic quenching [17]; similarly, the curve of $\left(F_{0}-F\right)^{-1}$ versus $Q^{-1}$ (Lineweaver-Burk curve) would be linear [41] for static quenching. If the quenching type is combined quenching (both static and dynamic), the Stern-Volmer plot is an upward curvature [42]. One way to distinguish dynamic from static quenching is to examine the

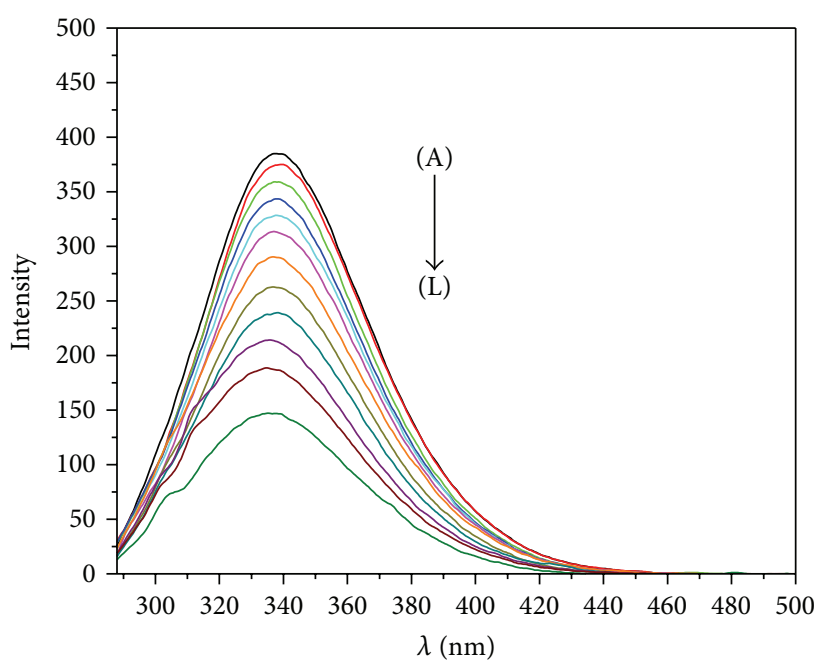

FIGURE 2: Fluorescence spectra of BSA $\left(50 \mu \mathrm{g} \cdot \mathrm{mL}^{-1}\right)$ in the absence (A) and in presence of EVG at different concentrations. EVG concentrations were $0.1 \mu \mathrm{g} \cdot \mathrm{mL}^{-1}$ (B), $0.3 \mu \mathrm{g} \cdot \mathrm{mL}^{-1}(\mathrm{C}), 0.5 \mu \mathrm{g} \cdot \mathrm{mL}^{-1}$ (D), $0.7 \mu \mathrm{g} \cdot \mathrm{mL}^{-1}$ (E), $1.0 \mu \mathrm{g} \cdot \mathrm{mL}^{-1}$ (F), $3.0 \mu \mathrm{g} \cdot \mathrm{mL}^{-1}$ (G), $5 \mu \mathrm{g} \cdot \mathrm{mL}^{-1}$ (H), $7.0 \mu \mathrm{g} \cdot \mathrm{mL}^{-1}(\mathrm{I}), 10 \mu \mathrm{g} \cdot \mathrm{mL}^{-1}(\mathrm{~J}), 12 \mu \mathrm{g} \cdot \mathrm{mL}^{-1}(\mathrm{~K})$, and $15 \mu \mathrm{g} \cdot \mathrm{mL}^{-1}$ (L).

temperature effect on the interaction of the drug to BSA. The Stern-Volmer and Lineweaver-Burk curves of EVG-BSA at different temperatures are shown in Figure 5. It can be seen that both Stern-Volmer and Lineweaver-Burk curves were linear, which illustrated that the quenching type was probably a static quenching.

Moreover, Table 1 demonstrates the gradual reduction of $K_{\mathrm{SV}}$ and $K_{\mathrm{LB}}$ values upon temperature increase that supports the idea of a static type quenching of BSA fluorescence [42]. This hypothesis was also backed up by the $K_{q}$ values (quenching rate constant), which was computed using the following equation

$$
K_{q}=\frac{K_{S V}}{\tau_{0}}
$$

Since the fluorescence lifetime of the biopolymer $\left(\tau_{0}\right)$ can be taken as $10^{-8} \mathrm{~s}^{-1}$ [43], then the $K_{q}$ values summarized in Table 1 were calculated for EVG-BSA system to be in the order of $10^{12} \mathrm{~L} \mathrm{Mol}^{-1} \mathrm{~s}^{-1}$. Previous reports have shown that the maximum scatter collision quenching constant, $K_{q}$ of various quenchers with the biopolymer is $2 \times 10^{10} \mathrm{~L} \mathrm{M}^{-1} \mathrm{~s}^{-1}$ [43]. Ultimately, the $K_{q}$ values of protein quenching procedure initiated by EVG are higher than the $K_{q}$ of the scattered procedure. This indicated that the quenching is not initiated by dynamic collision but from the formation of a complex [41].

3.2. Binding Constants and the Number of Binding Sites. Based upon the probability of a static quenching process is taking place between BSA and EVG and if it is assumed that there are independent binding sites to a set of equivalent sites on the 


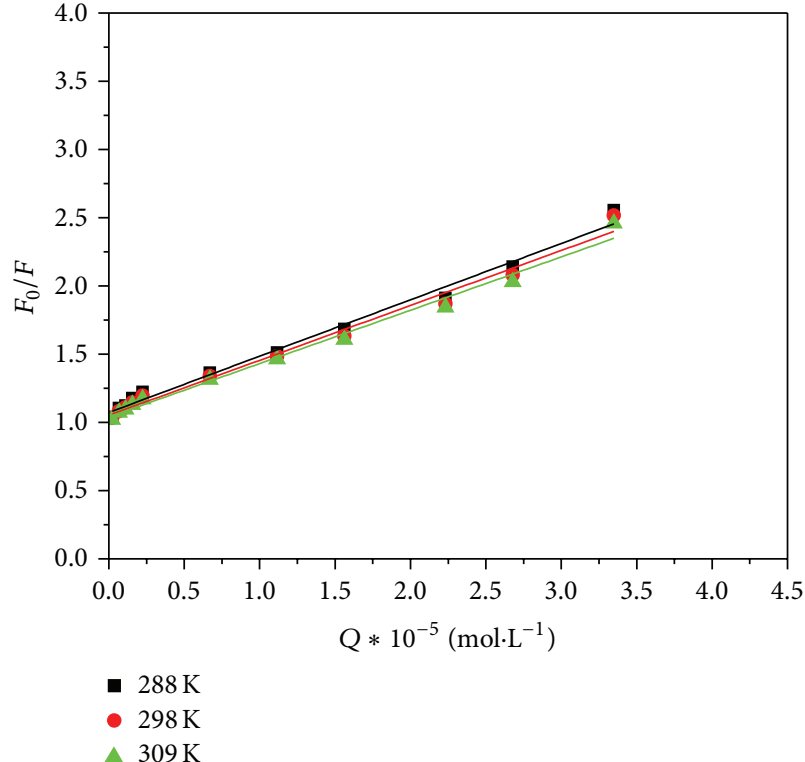

(a)

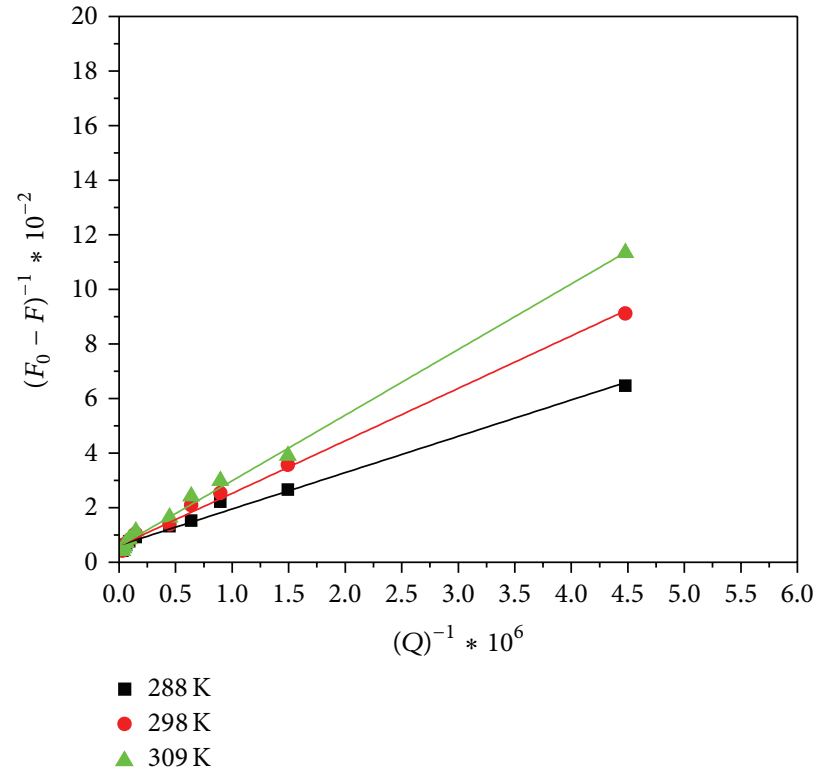

(b)

Figure 3: The Stern-Volmer (a) and Lineweaver-Burk (b) curves at different temperatures.

TABLE 1: Stern-Volmer and Lineweaver-Burk correlation parameters for BSA-EVG interaction.

\begin{tabular}{lcccr}
\hline $\begin{array}{l}\text { Temperature }(T) \\
(\mathrm{K})\end{array}$ & $K_{\mathrm{SV}} \times 10^{4}\left(\mathrm{~L} \mathrm{~mol}^{-1}\right)$ & $K_{q} \times 10^{12}\left(\mathrm{~L} \mathrm{~mol}^{-1} \mathrm{~s}^{-1}\right)$ & $r^{2}$ & \multicolumn{2}{c}{$K_{\mathrm{LB}} \times 10^{5}\left(\mathrm{~L} \mathrm{~mol}^{-1}\right)$} & 0.9949 \\
\hline 288 & $4.13 \pm 0.24$ & 4.13 & 0.9890 & $4.66 \pm 0.15$ \\
298 & $4.03 \pm 0.27$ & 4.03 & 0.9867 & $3.14 \pm 0.14$ \\
309 & $3.91 \pm 0.26$ & 3.91 & 0.9879 & $2.43 \pm 0.14$ \\
\hline
\end{tabular}

${ }^{*}$ Average of four determinations.

TABLE 2: Binding constant $K$ and the number of binding sites $n$.

\begin{tabular}{lcc}
\hline $\begin{array}{l}\text { Temperature }(T) \\
(\mathrm{K})\end{array}$ & $K \times 10^{3}\left(\mathrm{~L} \mathrm{~mol}^{-1}\right)$ & $n$ \\
\hline 288 & 2.68 & $0.67 \pm 0.03$ \\
298 & 1.99 & $0.72 \pm 0.02$ \\
309 & 1.12 & $0.75 \pm 0.03$ \\
\hline
\end{tabular}

protein, the apparent binding constant $(K)$ and the number of binding sites $(n)$ can be determined from $[42,44]$

$$
\log \left(\frac{F_{0}-F}{F}\right)=\log K+n \log Q
$$

where $K$ is the binding constant, reflecting the reaction degree of BSA and EVG; $n$ is the number of binding sites, specifying the number of EVG bound to a BSA molecule. Thus, a plot of $\log \left(F_{0}-F\right) / F$ versus $\log Q$ could be used to determine $K$ and $n$. The $K$ and $n$ at different temperatures are summarized in Table 2 , indicating good assumptions made for deriving (4). Table 2 also demonstrates a decrease in the binding constant with the increase in temperature, yielding a less stable EVG-BSA complex. Additionally, $n$ values were found to be $\sim 1$, which in turn may infer the involvement of one independent class of binding sites on BSA for EVG.

\subsection{Thermodynamics and Interaction Forces between EVG and} $B S A$. As the binding constant is dependent on temperature; hence, a thermodynamic process was regarded responsible for the complex formation. Therefore, temperature dependent thermodynamic parameters were determined for further investigating the acting forces between EVG and BSA. Generally, the force between a small molecule and a biological macromolecule includes hydrophobic interaction, hydrogen bonding, van der Waals force, and electrostatic force. The thermodynamic parameters associated with the different types of interactions were previously reported [45]. In which, hydrophobic force may increase $\Delta H^{\theta}$ and $\Delta S^{\theta}$ of a system, while van der Waals power may decrease them with negative $\Delta H^{\theta}$ indicative for hydrogen bonding and electrostatic force usually makes $\Delta H^{\theta} \sim 0$ and $\Delta S^{\theta}>0$ [46]. Thermodynamic parameters, enthalpy $\left(\Delta H^{\theta}\right)$, and entropy $\left(\Delta S^{\theta}\right)$ of EVGBSA interaction are significant to confirm binding mode. Binding studies were performed at 288, 298, and $309 \mathrm{~K}$, at which BSA does not undergo any structural degradation. 


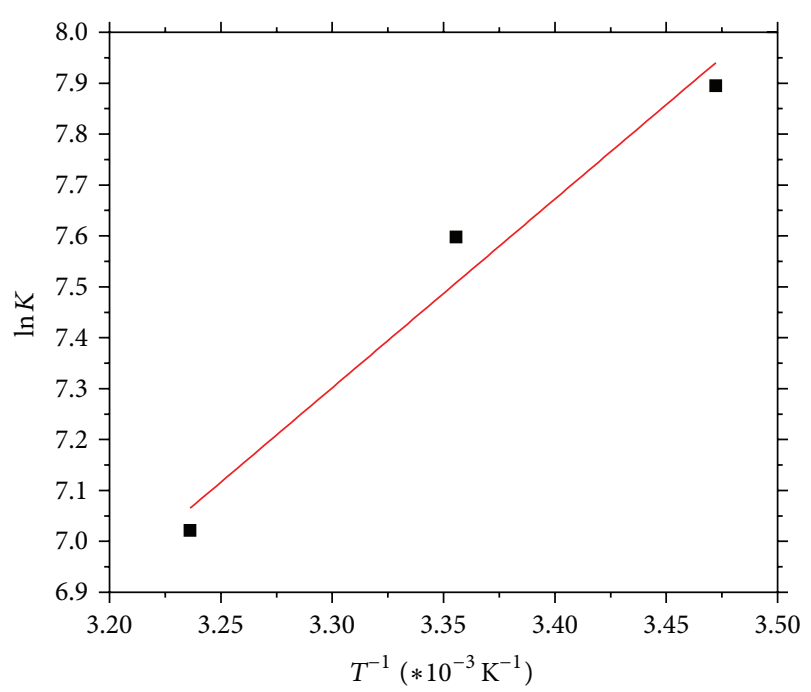

FIgURE 4: Van't Hoff plot for the binding between EVG and BSA, variation of $\ln K$ as a function of $1 / T$.

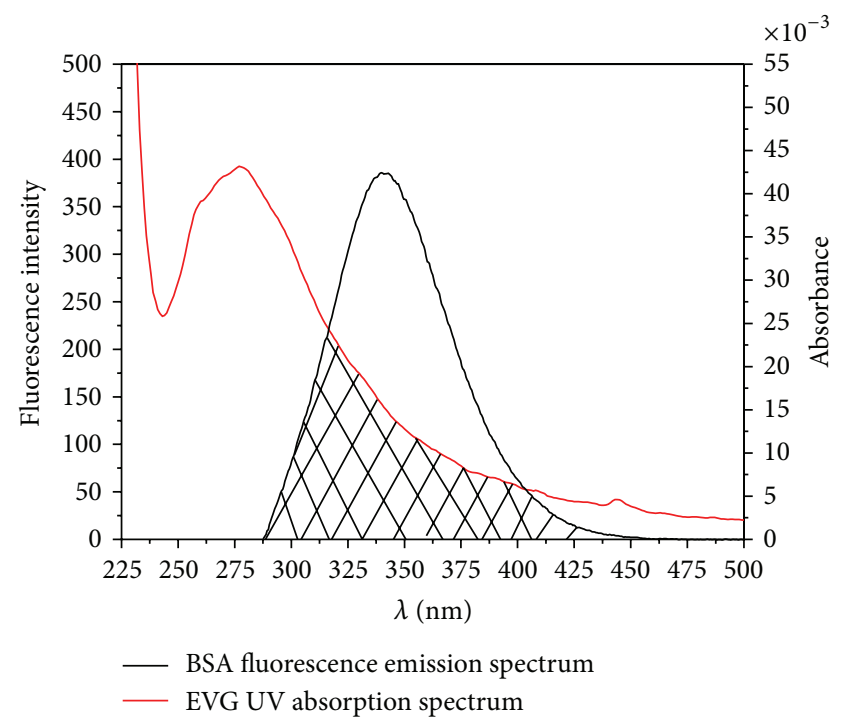

FIGURE 5: The overlap of the fluorescence spectrum of BSA (a) and the absorbance spectrum of EVG (b).

The thermodynamic parameters were evaluated using the Van't Hoff and Gibbs-Helmholtz [47, 48] equations:

$$
\begin{gathered}
\log K=\frac{-\Delta H^{\theta}}{2.303 R T}+\frac{\Delta S^{\theta}}{2.303 R} \\
\Delta G^{\theta}=\Delta H^{\theta}-T \cdot \Delta S^{\theta},
\end{gathered}
$$

where $K$ and $R$ are the binding constant and gas constant, respectively. $\Delta H^{\theta}, \Delta G^{\theta}$, and $\Delta S^{\theta}$ are enthalpy change, free energy change, and entropy change, respectively. Therefore, a plot of $\log K$ versus $1 / T$ (Figure 4 ) enables the determination of $\Delta H^{\theta}, \Delta G^{\theta}$, and $\Delta S^{\theta}$ by (5). Thermodynamic parameters for EVG-BSA binding are summarized in Table 3

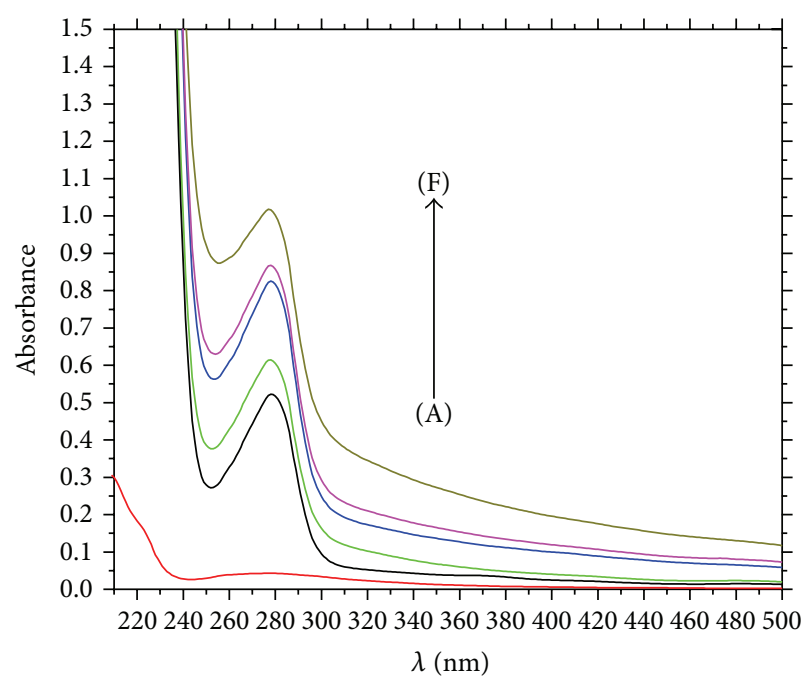

FIGURE 6: Absorbance spectra of EVG $10 \mu \mathrm{g} \mathrm{mL}^{-1}$ (A), BSA $0.8 \mathrm{mg} \mathrm{mL}^{-1}(\mathrm{~B})$, and EVG-BSA system at EVG concentration of $(\mathrm{C})$ $3.0 \mu \mathrm{g} \mathrm{mL}^{-1}$, (D) $5.0 \mu \mathrm{g} \mathrm{mL}^{-1}$, (E) $7.0 \mu \mathrm{g} \mathrm{mL}^{-1}$, and (F) $10 \mu \mathrm{g} \mathrm{mL}^{-1}$.

TABLE 3: Thermodynamic parameters of BSA-ELV binding.

\begin{tabular}{lccc}
\hline $\begin{array}{l}\text { Temperature }(T) \\
(\mathrm{K})\end{array}$ & $\begin{array}{c}\Delta G^{\theta} \\
(\mathrm{kJ} \mathrm{mol})\end{array}$ & $\begin{array}{c}\Delta H^{\theta} \\
\left(\mathrm{kJ} \mathrm{mol}^{-1}\right)\end{array}$ & $\begin{array}{c}\Delta S^{\theta} \\
\left(\mathrm{J} \mathrm{mol}^{-1} \mathrm{~K}^{-1}\right)\end{array}$ \\
\hline 288 & -42.64 & & \\
298 & -43.05 & -30.83 & 41.02 \\
309 & -43.50 & & \\
\hline
\end{tabular}

and demonstrate that hydrogen bonding and hydrophobic interactions play a role in the binding between EVG and BSA.

3.4. Energy Transfer between EVG and BSA. The aforementioned spectral studies have suggested that EVG forms a nonfluorescent complex with BSA. Three types of intrinsic fluorophores are known to be found in BSA sequence, namely, tryptophan (Trp), tyrosine (Tyr), and phenylalanine (Phe); however the intrinsic fluorescence of BSA is mainly due to tryptophan [49]. The efficiency of energy transfer can be used to determine the distance, $r$, between the drug and the tryptophan residues in the protein. The distance $r$ is an average value between the bound EVG and the two tryptophan residues of BSA (Trp-135 and Trp-214) and can be determined using Förster's resonance energy transfer (FRET) $[35,44]$. Generally, energy transfer occurs whenever the emission spectrum of a fluorophore (donor) overlaps with the absorption spectrum of another molecule (acceptor) and the distance between them is less than $8 \mathrm{~nm}$. The overlap of the UV absorption spectrum of EVG with the fluorescence emission spectra of BSA is shown in Figure 5. The efficiency of energy transfer, $E$, is calculated using

$$
E=1-\frac{F}{F_{0}}=\frac{R_{0}^{6}}{R_{0}^{6}+r^{6}},
$$

where $F$ and $F_{0}$ are the fluorescence intensities of BSA in the presence and absence of EVG, $r$ is the distance between 


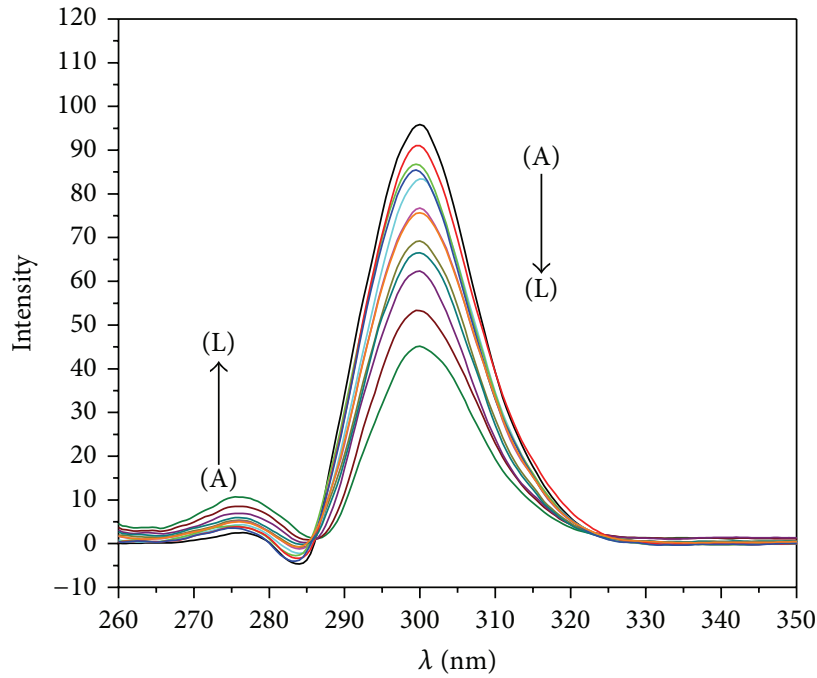

(a)

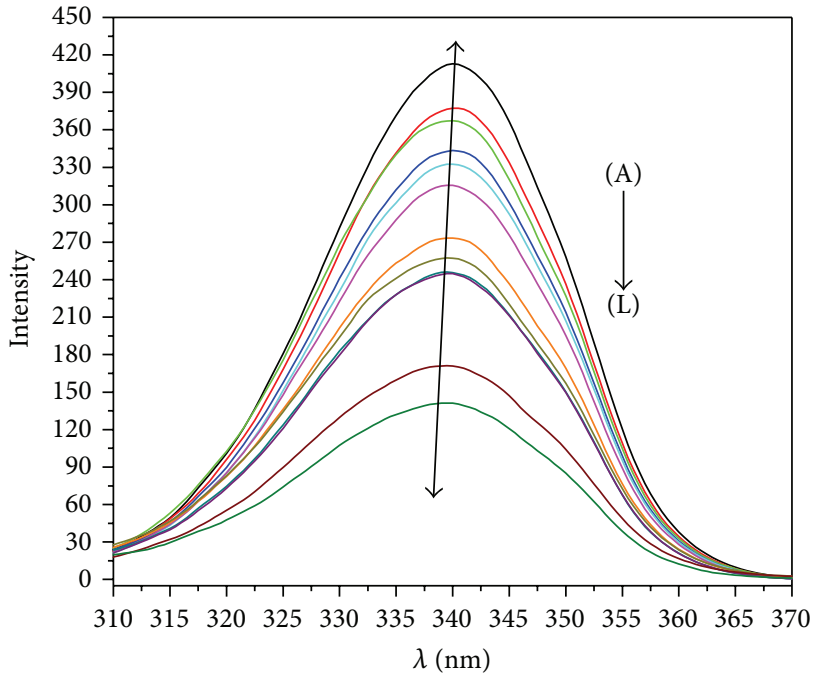

(b)

FIGURE 7: Effect of EVG on the synchronous fluorescence spectra of BSA with $\Delta \lambda=15 \mathrm{~nm}(\mathrm{~A})$ and $\Delta \lambda=60 \mathrm{~nm}(\mathrm{~B}) . c \mathrm{BSA}=50 \mu \mathrm{g} \cdot \mathrm{mL}^{-1}, c \mathrm{EVG}$ $(A-\mathrm{L})=\left(0,0.3,0.7,1.0,3.0,5.0,10,12\right.$, and $\left.15 \mu \mathrm{g} \cdot \mathrm{mL}^{-1}\right)$.

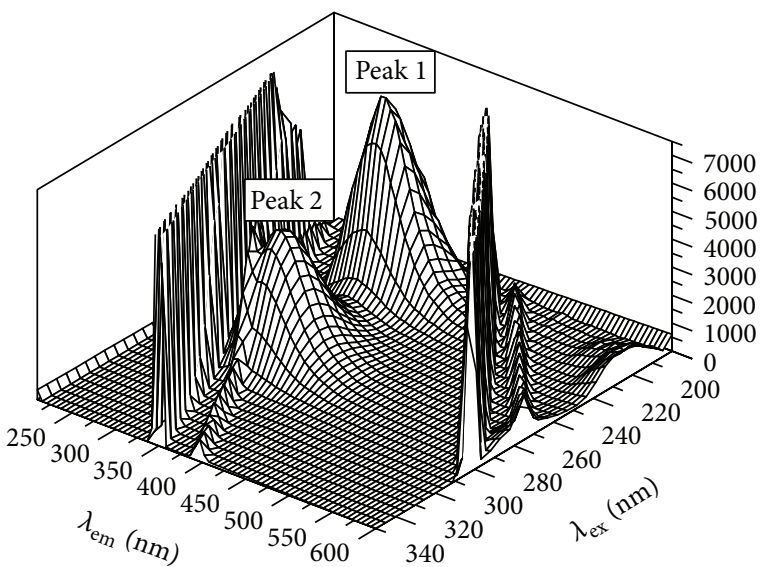

(a)

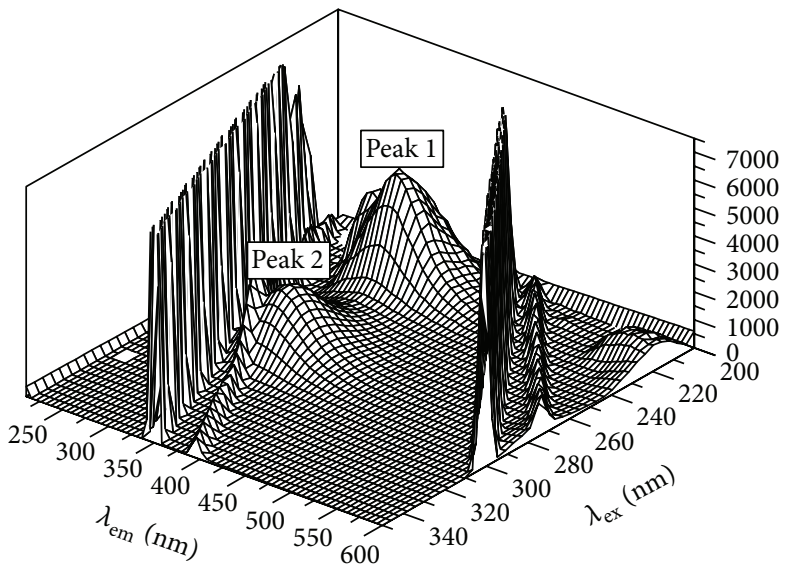

(b)

FIGURE 8: Three-dimensional spectra of BSA $\left(50 \mu \mathrm{g} \cdot \mathrm{mL}^{-1}\right)$ in the absence (a) and presence (b) of EVG $\left(10 \mu \mathrm{g} \cdot \mathrm{mL}^{-1}\right)$.

acceptor and donor and $R_{0}$ is the critical distance when the transfer efficiency is $50 \%$ :

$$
R_{0}^{6}=8.8 \times 10^{-25} k^{2} N^{-4} \phi J
$$

where $k^{2}$ is the spatial orientation factor of the dipole, $N$ is the refractive index of the medium, $\Phi$ is the fluorescence quantum yield of the donor, and $J$ is the overlap integral of the fluorescence emission spectrum of the donor and the absorption spectrum of the acceptor. $J$ is given by

$$
J=\frac{\int_{0}^{\infty} F(\lambda) \varepsilon(\lambda) \lambda^{4} \Delta \lambda}{\int_{0}^{\infty} F(\lambda) \Delta \lambda},
$$

where $F(\lambda)$ is the fluorescence intensity of the fluorescent donor of wavelength, $\lambda$, and $\varepsilon(\lambda)$ is the molar absorption coefficient of the acceptor at wavelength, $\lambda$. In the current study, values of $k^{2}=2 / 3, N=1.336$, and $\Phi=0.15$ were used as reported in the literature [50]. Hence, values of $J=1.85 \times$ $10^{-17} \mathrm{~cm}^{3} \mathrm{~L} \mathrm{~mol}^{-1}, R_{0}=1.95 \mathrm{~nm}, E=0.18$, and $r=2.51 \mathrm{~nm}$ were calculated. The donor-to-acceptor distance, $r<8 \mathrm{~nm}$ $[50,51]$, indicated that the energy transfer from BSA to EVG occurs with high possibility. Larger BSA-EVG distance, $r$ compared to that of $R_{0}$ values observed in this study, also reveals the presence of static type quenching mechanism $[52,53]$

\subsection{Conformation Investigation}

3.5.1. UV-Vis Absorption Spectra. The complex formation between EVG-BSA was also evident from UV-Vis absorption spectral data (Figure 6). It is obvious that the UV absorption 


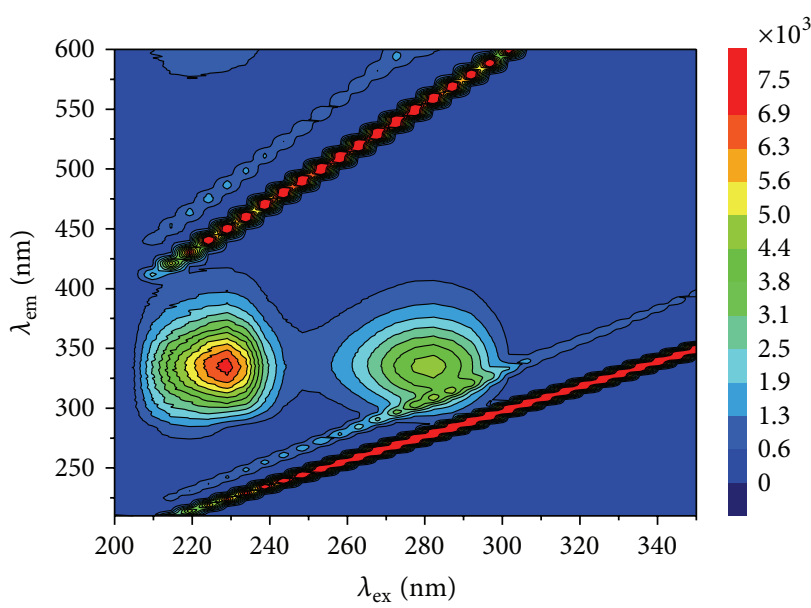

(a)

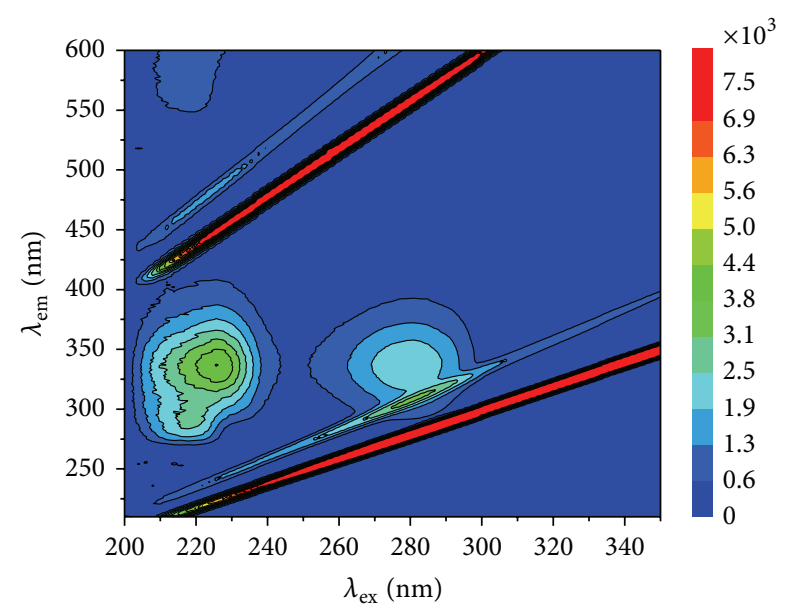

(b)

FIGURE 9: Contour fluorescence intensity spectra of BSA (a) and EVG-BSA system (b).

intensity of BSA increased regularly with the variation of EVG concentration, indicating that the peptide strands of BSA molecules extended more upon the addition of EVG [51].

\subsubsection{Synchronous Fluorescence. The synchronous fluores-} cence spectra were measured, shown in Figure 7. In the synchronous spectra, the sensitivity associated with fluorescence is maintained while offering several advantages: spectral simplification, spectral bandwidth reduction, and avoidance of different perturbing effects. It shows the tyrosine residues (Tyr) and tryptophan residues (Trp) of BSA when the wavelength interval $\Delta \lambda$ is $15 \mathrm{~nm}$ and $\Delta \lambda$ is $60 \mathrm{~nm}$, respectively [54, 55]. It is obvious that the emission strengths of both tyrosine and tryptophan decreased. A slight blue shift can be observed in Figure 7(b) which indicates that the polarity around tryptophan residue decreased. That is to say, tryptophan residues were placed in a more hydrophobic environment and less exposed to the solvent. Additionally, it can be seen from Figure 7 that a stronger fluorescence quenching effect of tryptophan residues compared with the tyrosine residues after EVG is added. It indicates that the binding site of EVG may be closer to tryptophan than that of the tyrosine residues.

3.5.3. Three-Dimensional Fluorescence. Moreover, threedimensional (3D) fluorescence spectra were recorded, and some characteristic 3D parameters are reported in Table 4. Two typical fluorescence peaks can easily be observed in the three-dimensional fluorescence spectra Figures 8 and 9. The peak at $(224 \rightarrow 333)$ (Peak 1) mainly exhibits the fluorescence characteristic of $n \rightarrow \pi^{*}$ transition of the polypeptide backbone of protein structure, $\mathrm{C}=\mathrm{O}$ [56]. The second peak at $(280 \rightarrow 337)$ (Peak 2) revealed the spectral characteristic of Trp and Tyr residues [19]. The Rayleigh scattering peaks and two typical fluorescence peaks can be easily observed in 3D fluorescence contour map of BSA
TABLE 4: Three-dimensional fluorescence characteristic parameters of EVG-BSA binding.

\begin{tabular}{lcccc}
\hline & \multicolumn{2}{c}{ BSA } & \multicolumn{2}{c}{ EVG-BSA } \\
& Peak 1 & Peak 2 & Peak 1 & Peak 2 \\
\hline $\begin{array}{l}\text { Peak position } \\
(\lambda \text { ex/ } \text { em, nm/nm) }\end{array}$ & $224 / 333$ & $280 / 337$ & $224 / 336.5$ & $280 / 336$ \\
$\begin{array}{l}\text { Relative intensity } \\
(\text { IF })\end{array}$ & 7352.52 & 4700.98 & 4426.11 & 2491.21 \\
$\begin{array}{l}\text { Stokes shift } \\
(\Delta \lambda / \mathrm{nm})\end{array}$ & 109 & 57 & 112.5 & 56 \\
\hline
\end{tabular}

(Figure 9). It is clear that both BSA's fluorescence peaks were quenched by the addition of EVG, differing in their extents (in the presence of EVG, the intensity ratio of the fluorescence peak to the second order one is $1.776: 1$ whereas it is $1.564: 1$ in the absence of EVG). Additionally, Rayleigh scattering peaks were enhanced that also give clear evidence of complex formation between EVG and BSA [57].

\section{Conclusions}

In this work we have employed a set of fluorescence spectroscopic UV-visible spectrophotometric analyses to study the interactions of BSA with EVG. EVG proved to bind to BSA resulting in reduction of its fluorescence intensity via static binding. We have computed the binding parameters, and conformational changes leading to increased stability of EVG-BSA complex. Data analysis showed that only one binding site on BSA is available for EVG with a binding constant in the order of $10^{3} \mathrm{~L} \mathrm{~mol}^{-1}$. Thermodynamic parameters were also computed to be negativefree energy and enthalpy while positive entropy referring a spontaneous reaction of BSA with EVG with both hydrogen bonding and hydrophobic interactions probably involved in the binding. Conformation study showed that the polarity around tryptophan residue 
decreased. This demonstrates that tryptophan residues were placed in a more hydrophobic microenvironment and less exposed to the solvent. The binding distance between the donor (BSA) and the acceptor (EVG) was calculated based on the Förster's theory of nonradiation energy transfer.

\section{Conflict of Interests}

The author declares that there is no conflict of interests regarding the publication of this paper.

\section{Acknowledgment}

The authors would like to extend their sincere appreciation to the Deanship of Scientific Research at King Saud University for its funding this Research Group no. RG-1435-025.

\section{References}

[1] A. B. Khan, J. M. Khan, M. S. Ali, R. H. Khan, and D. KabirUd, "Interaction of amphiphilic drugs with human and bovine serum albumins," Spectrochimica Acta Part A: Molecular and Biomolecular Spectroscopy, vol. 97, pp. 119-124, 2012.

[2] X. M. He and D. C. Carter, "Atomic structure and chemistry of human serum albumin," Nature, vol. 358, no. 6383, pp. 209-215, 1992.

[3] U. Kragh-Hansen, "Molecular aspects of ligand binding to serum albumin," Pharmacological Reviews, vol. 33, no. 1, pp. 1753, 1981.

[4] A. Varshney, P. Sen, E. Ahmad, M. Rehan, N. Subbarao, and R. H. Khan, "Ligand binding strategies of human serum albumin: how can the cargo be utilized?" Chirality, vol. 22, no. 1, pp. 77-87, 2010.

[5] S. K. Chaturvedi, E. Ahmad, J. M. Khan, P. Alam, M. Ishtikhar, and R. H. Khan, "Elucidating the interaction of limonene with bovine serum albumin: a multi-technique approach," Molecular BioSystems, vol. 11, no. 1, pp. 307-316, 2015.

[6] P. Sen, B. Ahmad, and R. H. Khan, "Formation of a molten globule like state in bovine serum albumin at alkaline $\mathrm{pH}$," European Biophysics Journal, vol. 37, no. 8, pp. 1303-1308, 2008.

[7] P. Bolel, N. Mahapatra, and M. Halder, "Optical spectroscopic exploration of binding of cochineal red a with two homologous serum albumins," Journal of Agricultural and Food Chemistry, vol. 60, no. 14, pp. 3727-3734, 2012.

[8] D. C. Carter and J. X. Ho, "Structure of serum albumin," Advances in Protein Chemistry, vol. 45, pp. 153-203, 1994.

[9] A. A. Bhattacharya, T. Grüne, and S. Curry, "Crystallographic analysis reveals common modes of binding of medium and long-chain fatty acids to human serum albumin," Journal of Molecular Biology, vol. 303, no. 5, pp. 721-732, 2000.

[10] A. A. Spector, K. John, and J. E. Fletcher, "Binding of longchain fatty acids to bovine serum albumin," The Journal of Lipid Research, vol. 10, no. 1, pp. 56-67, 1969.

[11] I. E. Borissevitch, T. T. Tominaga, H. Imasato, and M. Tabak, "Fluorescence and optical absorption study of interaction of two water soluble porphyrins with bovine serum albumin. The role of albumin and porphyrin aggregation," Journal of Luminescence, vol. 69, no. 2, pp. 65-76, 1996.

[12] G. Blauer and T. E. King, "Interactions of bilirubin with bovine serum albumin in aqueous solution," Journal of Biological Chemistry, vol. 245, no. 2, pp. 372-381, 1970.
[13] J. Tian, J. Liu, Z. Hu, and X. Chen, "Interaction of wogonin with bovine serum albumin," Bioorganic and Medicinal Chemistry, vol. 13, no. 12, pp. 4124-4129, 2005.

[14] N. Zhou, Y.-Z. Liang, and P. Wang, " $18 \beta$-Glycyrrhetinic acid interaction with bovine serum albumin," Journal of Photochemistry and Photobiology A: Chemistry, vol. 185, no. 2-3, pp. 271276, 2007.

[15] Y.-Q. Wang, H.-M. Zhang, G.-C. Zhang, W.-H. Tao, Z.-H. Fei, and Z.-T. Liu, "Spectroscopic studies on the interaction between silicotungstic acid and bovine serum albumin," Journal of Pharmaceutical and Biomedical Analysis, vol. 43, no. 5, pp. 1869-1875, 2007.

[16] Y. P. Wang, Y. L. Wei, and C. Dong, "Study on the interaction of 3, 3-bis(4-hydroxy-1-naphthyl)-phthalide with bovine serum albumin by fluorescence spectroscopy," Journal of Photochemistry and Photobiology A: Chemistry, vol. 177, pp. 6-11, 2006.

[17] A. Gong, X. Zhu, Y. Hu, and S. Yu, "A fluorescence spectroscopic study of the interaction between epristeride and bovin serum albumine and its analytical application," Talanta, vol. 73, no. 4, pp. 668-673, 2007.

[18] B. Zhou, Z.-D. Qi, Q. Xiao, J.-X. Dong, Y.-Z. Zhang, and Y. Liu, "Interaction of loratadine with serum albumins studied by fluorescence quenching method," Journal of Biochemical and Biophysical Methods, vol. 70, no. 5, pp. 743-747, 2007.

[19] A. Sułkowska, "Interaction of drugs with bovine and human serum albumin," Journal of Molecular Structure, vol. 614, no. 1-3, pp. 227-232, 2002.

[20] N. Gull, P. Sen, and R. H. Khan, "Interaction of bovine (BSA), rabbit (RSA), and porcine (PSA) serum albumins with cationic single-chain/gemini surfactants: a comparative study," Langmuir, vol. 25, no. 19, pp. 11686-11691, 2009.

[21] N. Zaidi, S. Nusrat, F. K. Zaidi, and R. H. Khan, "pH-dependent differential interacting mechanisms of sodium dodecyl sulfate with bovine serum fetuin: a biophysical insight," The Journal of Physical Chemistry B, vol. 118, no. 46, pp. 13025-13036, 2014.

[22] E. J. Arts and D. J. Hazuda, "HIV-1 antiretroviral drug therapy," Cold Spring Harbor Perspectives in Medicine, vol. 2, no. 4, Article ID a007161, 2012.

[23] S. L. Karmon and M. Markowitz, "Next-generation integrase inhibitors: where to after raltegravir?" Drugs, vol. 73, no. 3, pp. 213-228, 2013.

[24] S. Ramanathan, A. A. Mathias, P. German, and B. P. Kearney, "Clinical pharmacokinetic and pharmacodynamic profile of the HIV integrase inhibitor elvitegravir," Clinical Pharmacokinetics, vol. 50, no. 4, pp. 229-244, 2011.

[25] K. Shimura, E. Kodama, Y. Sakagami et al., "Broad antiretroviral activity and resistance profile of the novel human immunodeficiency virus integrase inhibitor elvitegravir (JTK-303/GS9137)," Journal of Virology, vol. 82, no. 2, pp. 764-774, 2008.

[26] J. Marinello, C. Marchand, B. T. Mott, A. Bain, C. J. Thomas, and Y. Pommier, "Comparison of raltegravir and elvitegravir on HIV-1 integrase catalytic reactions and on a series of drugresistant integrase mutants," Biochemistry, vol. 47, no. 36, pp. 9345-9354, 2008.

[27] A. R. Zolopa, D. S. Berger, H. Lampiris et al., "Activity of elvitegravir, a once-daily integrase inhibitor, against resistant HIV type 1: results of a phase 2, randomized, controlled, doseranging clinical trial," The Journal of Infectious Diseases, vol. 201, no. 6, pp. 814-822, 2010.

[28] E. D. Deeks, "Elvitegravir: a review of its use in adults with HIV1 infection," Drugs, vol. 74, no. 6, pp. 687-697, 2014. 
[29] Gilead Sciences International, Vitekta Film-Coated Tablets: EU Summary of Product Characteristics, Gilead Sciences International, 2013, http://ec.europa.eu/health/documents/community-register/2013/20131113126981/anx_126981_en.pdf.

[30] U.S. Food and Drug Administration (FDA), Approval of Vitekta, 2014, http://www.accessdata.fda.gov/drugsatfda_docs/label/2014/ 203093s000lbl.pdf.

[31] S. Sugio, A. Kashima, S. Mochizuki, M. Noda, and K. Kobayashi, "Crystal structure of human serum albumin at $2.5 \AA$ resolution," Protein Engineering, vol. 12, no. 6, pp. 439-446, 1999.

[32] I. Petitpas, T. Grüne, A. A. Bhattacharya, and S. Curry, "Crystal structures of human serum albumin complexed with monounsaturated and polyunsaturated fatty acids," Journal of Molecular Biology, vol. 314, no. 5, pp. 955-960, 2001.

[33] H.-X. Zhang, S. Gao, and X.-X. Yang, "Synthesis of an octupolar compound and its biological effects on serum albumin," Molecular Biology Reports, vol. 36, no. 6, pp. 1405-1411, 2009.

[34] P. B. Kandagal, J. Seetharamappa, S. Ashoka, S. M. T. Shaikh, and D. H. Manjunatha, "Study of the interaction between doxepin hydrochloride and bovine serum albumin by spectroscopic techniques," International Journal of Biological Macromolecules, vol. 39, no. 4-5, pp. 234-239, 2006.

[35] Th. Förster, "Zwischenmolekulare Energiewanderung und Fluoreszenz," Annalen der Physik, vol. 437, no. 1-2, pp. 55-75, 1948.

[36] J. R. Lakowicz, Principle of Fluorescence Spectroscopy, Plemum Press, New York, NY, USA, 2nd edition, 1999.

[37] H. Wang, J. Mao, A. Duan et al., "Fluorescence quenching of 4-tert-octylphenol by room temperature ionic liquids and its application," Journal of Fluorescence, vol. 23, no. 2, pp. 323-331, 2013.

[38] G.-Z. Chen, X.-Z. Huang, and J.-G. Xu, Spectrofluorimetric Analytical Method, Science Press, Beijing, China, 2nd edition, 1990.

[39] O. Stern and M. Volmer, "The extinction period of fluorescence," Physikalische Zeitschrift, vol. 20, pp. 183-188, 1919.

[40] H. Lineweaver and D. Burk, "The determination of enzyme dissociation constants," Journal of the American Chemical Society, vol. 56, no. 3, pp. 658-666, 1934.

[41] W. R. Ware, "Oxygen quenching of fluorescence in solution: an experimental study of the diffusion process," The Journal of Physical Chemistry, vol. 66, no. 3, pp. 455-458, 1962.

[42] Y. Huang, Z. Zhang, D. Zhang, and J. Lv, "Flow-injection analysis chemiluminescence detection combined with microdialysis sampling for studying protein binding of drug," Talanta, vol. 53, no. 4, pp. 835-841, 2001.

[43] J. R. Lakowicz and G. Weber, "Quenching of fluorescence by oxygen. A probe for structural fluctuations in macromolecules," Biochemistry, vol. 12, no. 21, pp. 4161-4170, 1973.

[44] J. Liu, J.-N. Tian, J. Zhang, Z. Hu, and X. Chen, "Interaction of magnolol with bovine serum albumin: a fluorescencequenching study," Analytical and Bioanalytical Chemistry, vol. 376, no. 6, pp. 864-867, 2003.

[45] T. Forster and O. Sinanoglu, Modern Quantum Chemistry, vol. 3, Academic Press, New York, NY, USA, 1996.

[46] P. D. Ross and S. Subramanian, "Thermodynamics of protein association reactions: forces contributing to stability," Biochemistry, vol. 20, no. 11, pp. 3096-3102, 1981.

[47] H.-X. Zhang, X. Huang, and M. Zhang, "Thermodynamic studies on the interaction of dioxopromethazine to $\beta$-cyclodextrin and bovine serum albumin," Journal of Fluorescence, vol. 18, no. 3-4, pp. 753-760, 2008.
[48] H. D. B. Jenkins, "Gibbs-Helmholtz equation," in Chemical Thermodynamics at a Glance, pp. 150-153, Blackwell Publishing, 2008.

[49] Z. Jannesari, H. Hadadzadeh, T. Khayamian, B. Maleki, and H. A. Rudbari, "Experimental and molecular modeling studies on the interaction of the $\mathrm{Ru}(\mathrm{II})$-piroxicam with DNA and BSA," European Journal of Medicinal Chemistry, vol. 69, pp. 577-590, 2013.

[50] B. Valeur and J. C. Brochon, New Trends in Fluorescence Spectroscopy, Springer Press, Berlin, Germany, 6th edition, 1999.

[51] Y.-J. Hu, Y. Liu, J.-B. Wang, X.-H. Xiao, and S.-S. Qu, "Study of the interaction between monoammonium glycyrrhizinate and bovine serum albumin," Journal of Pharmaceutical and Biomedical Analysis, vol. 36, no. 4, pp. 915-919, 2004.

[52] W. He, Y. Li, C. Xue, Z. Hu, X. Chen, and F. Sheng, "Effect of Chinese medicine alpinetin on the structure of human serum albumin," Bioorganic \& Medicinal Chemistry, vol. 13, no. 5, pp. 1837-1845, 2005.

[53] F.-L. Cui, J. Fan, J.-P. Li, and Z.-D. Hu, "Interactions between 1benzoyl-4-p-chlorophenyl thiosemicarbazide and serum albumin: investigation by fluorescence spectroscopy, Bioorganic \& Medicinal Chemistry, vol. 12, no. 1, pp. 151-157, 2004.

[54] H. Yan, S. Zhao, J. Yang et al., "Interaction between levamisole hydrochloride and bovine serum albumin and the influence of alcohol: spectra," Journal of Solution Chemistry, vol. 38, no. 9, pp. 1183-1192, 2009.

[55] H.-X. Zhang, X. Huang, P. Mei, and S. Gao, "Interaction between glyoxal-bis-(2-hydroxyanil) and bovine serum albumin in solution," Journal of Solution Chemistry, vol. 37, no. 5, pp. 631-640, 2008.

[56] A. N. Glazer and E. L. Smith, "Studies on the ultraviolet difference spectra of proteins and polypeptides," The Journal of Biological Chemistry, vol. 236, pp. 2942-2947, 1961.

[57] M. Ishtikhar, S. Khan, G. Badr, A. Osama Mohamed, and R. Hasan Khan, "Interaction of the 5-fluorouracil analog 5-fluoro2 '-deoxyuridine with ' $\mathrm{N}$ ' and ' $\mathrm{B}$ ' isoforms of human serum albumin: a spectroscopic and calorimetric study," Molecular BioSystems, vol. 10, no. 11, pp. 2954-2964, 2014. 

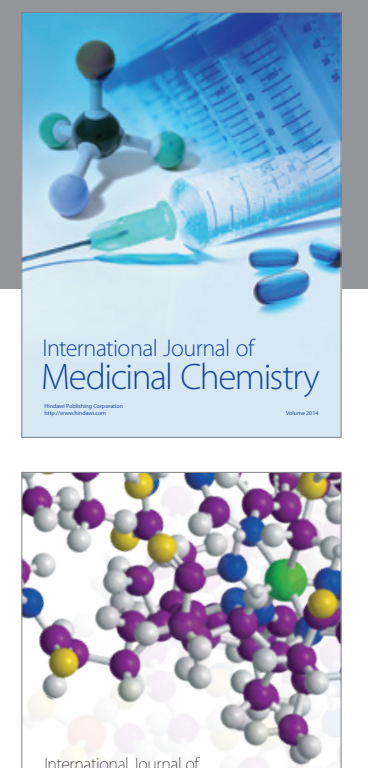

\section{Carbohydrate} Chemistry

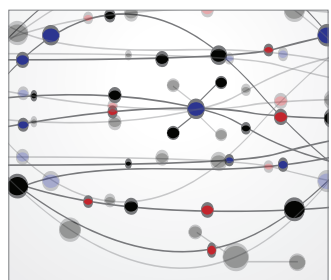

The Scientific World Journal
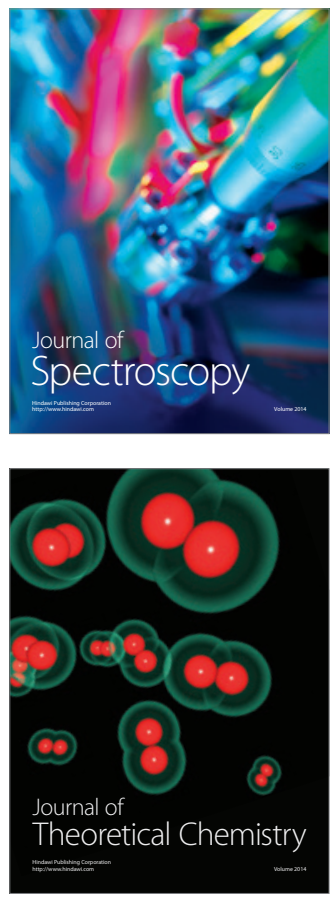
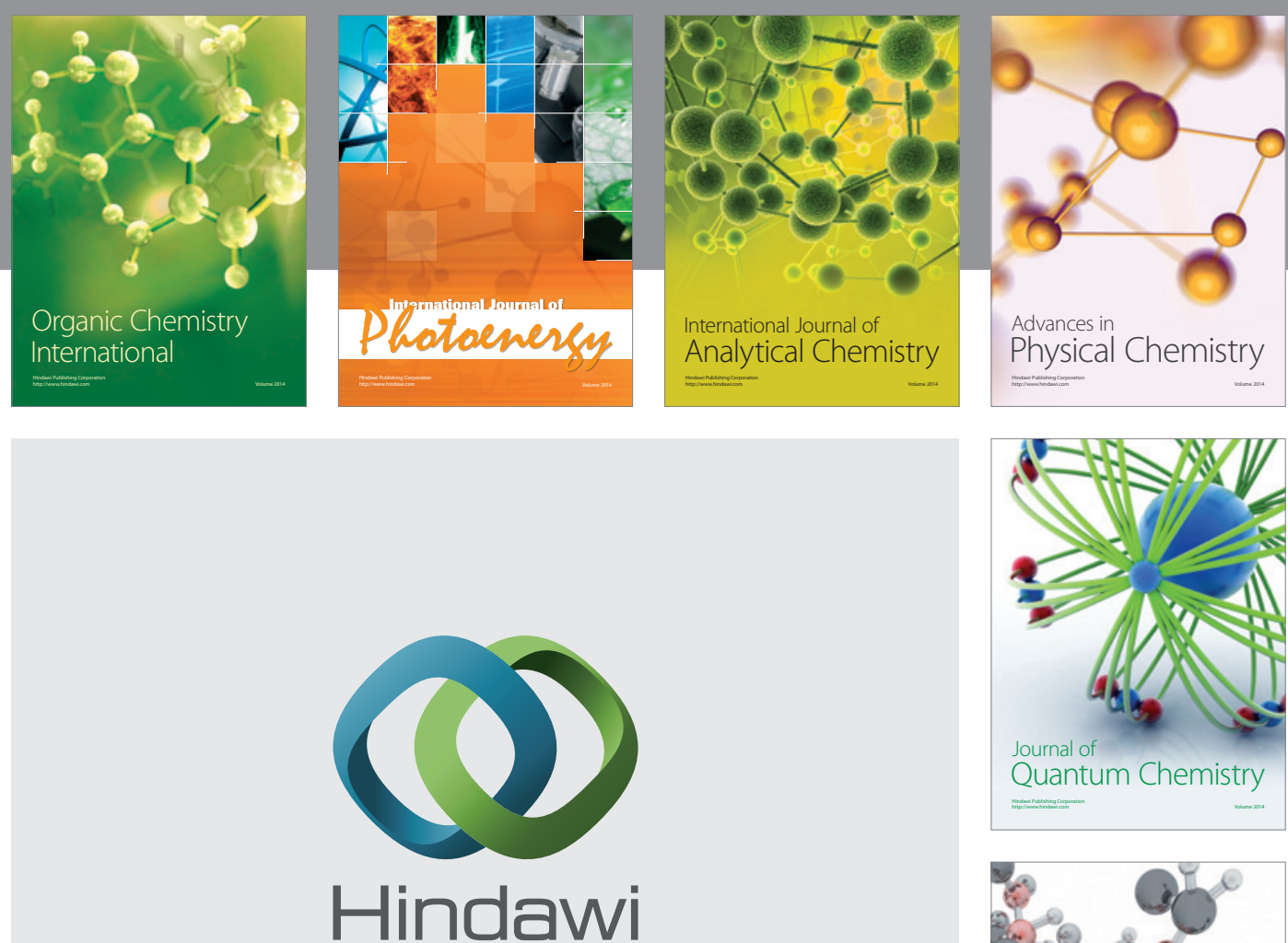

Submit your manuscripts at

http://www.hindawi.com



Analytical Methods

in Chemistry

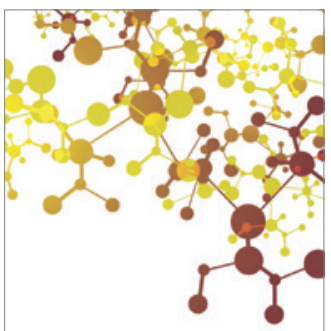

Journal of

Applied Chemistry

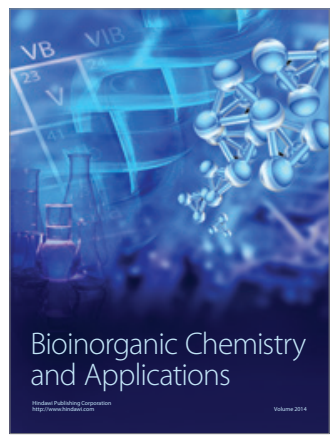

Inorganic Chemistry
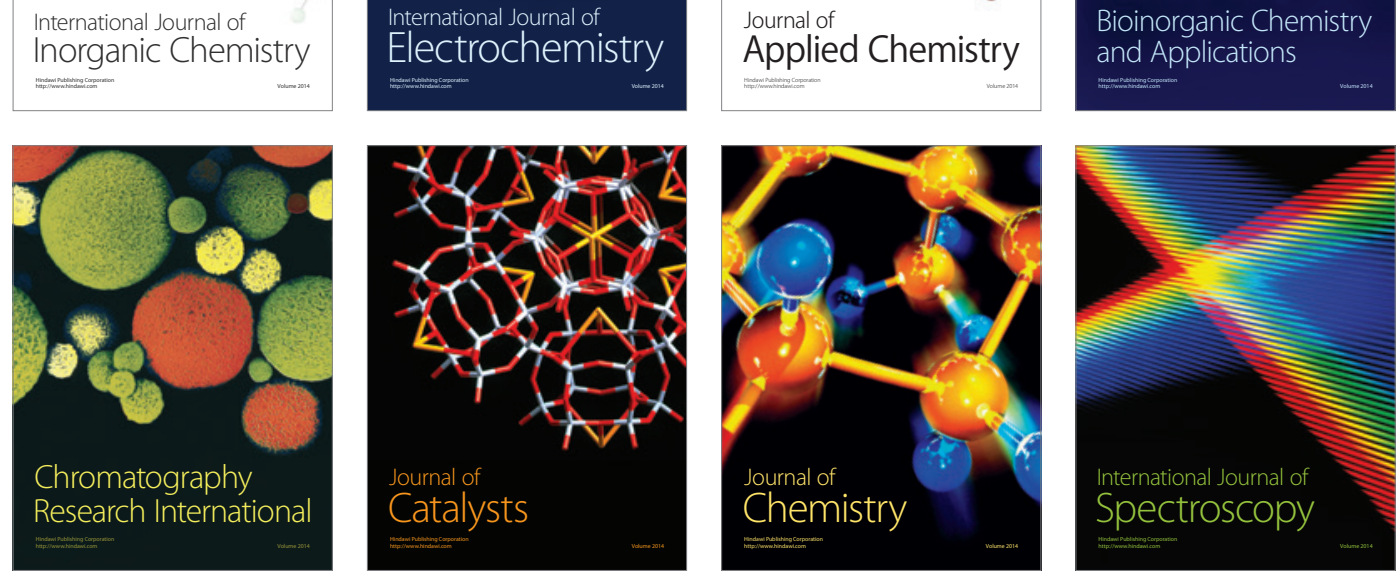